Commun. Korean Math. Soc. 21 (2006), No. 1, pp. 89-100

\title{
COMMON FIXED POINTS OF COMPATIBLE MAPS OF TYPE $(\beta)$ ON FUZZY METRIC SPACES
}

\author{
Servet Kutukcu, Duran Turkoglu, and Cemil Yildiz
}

\begin{abstract}
In this paper we prove a common fixed point theorem for compatible maps of type $(\beta)$ on fuzzy metric spaces with arbitrary continuous t-norm.
\end{abstract}

\section{Introduction}

The notion of fuzzy sets was introduced by Zadeh [28]. Deng [4], Erceg [6], Kaleva and Seikkala [15] and Kramosil and Michalek [18] have introduced the concepts of fuzzy metric spaces in different ways. George and Veeramani [8] modified the concept of fuzzy metric spaces introduced by Kramosil and Michalek [18] in order to get the Hausdorff topology.

Grebiec [9] extended the fixed point theorems of Banach [1] and Edelstein [5] to fuzzy metric spaces in the sense of Kramosil and Michalek [18] whose study is useful in the field of fixed point theorems of contractive type maps. Since then Fang [7] proved some fixed point theorems in fuzzy metric spaces, which improve, generalize and extend some main results of $[1,5,10-12,23]$.

Sessa [24] defined a generalization of commutativity, which called weak commutativity. Further Jungck [14] introduced more generalized commutativity, so called compatibility. Following Grabiec [9], Kramosil and Michalek [18] and Mishra et al. [19] obtained common fixed point theorems for compatible maps and asymptotically commuting maps on fuzzy metric spaces which generalize, extend and fuzzify several fixed point theorems for contractive-type maps on metric spaces and other spaces.

Received June 10, 2005.

2000 Mathematics Subject Classification: Primary 54H25; Secondary 47H10.

Key words and phrases: fuzzy metric spaces, common fixed point, compatible maps of type $(\beta)$. 
Pathak et al. [20] introduced the concept of compatible maps of type $(P)$ in metric spaces, which is equivalent to the concept of compatible maps under some conditions and proved common fixed point theorems in metric spaces. Cho et al. [3] introduced the notion of compatible maps of type $(\beta)$ in fuzzy metric spaces.

Many authors have studied the fixed point theory in fuzzy metric spaces. The most interesting references are [7, 9-11, 19, 21, 25].

In this paper, we prove common fixed point theorems for four maps satisfying some conditions in fuzzy metric spaces in the sense of George and Veeramani [8]. We also give an example to illustrate our main theorem.

\section{Preliminaries}

Now, we give some definitions.

Definition 1. (Schweizer and Sklar [22]). A binary operation * : $[0,1] \times[0,1] \rightarrow[0,1]$ is called a continuous t-norm if $([0,1], *)$ is an Abelian topological monoid with the unit 1 such that $a * b \leq c * d$ whenever $a \leq c$ and $b \leq d$ for all $a, b, c, d \in[0,1]$.

Examples of t-norms are $a * b=a b$ and $a * b=\min \{a, b\}$.

Definition 2. (George and Veeramani [8]). The 3-tuble $(X, M, *)$ is called a fuzzy metric space (shortly FM-space) if $X$ is an arbitrary set, * is a continuous t-norm and $M$ is a fuzzy set in $X^{2} \times[0, \infty)$ satisfying the following conditions: for all $x, y, z \in X$ and $t, s>0$,

(fm-1): $M(x, y, t)>0$,

(fm-2): $M(x, y, t)=1$ for all $t>0$ if and only if $x=y$,

(fm-3): $M(x, y, t)=M(y, x, t)$,

(fm-4): $M(x, y, t) * M(y, z, s) \leq M(x, z, t+s)$,

$(\mathbf{f m}-5): M(x, y,):.[0, \infty) \rightarrow[0,1]$ is continuous.

Definition 3. (Grabiec [9]). Let $(X, M, *)$ be an FM-space:

(1) A sequence $\left\{x_{n}\right\}$ in $X$ is said to be convergent to a point $x$ in $X$ (denoted by $\lim _{n \rightarrow \infty} x_{n}=x$ ) if $\lim _{n \rightarrow \infty} M\left(x_{n}, x, t\right)=1$ for all $t>0$.

(2) A sequence $\left\{x_{n}\right\}$ in $X$ is called a Cauchy sequence if

$$
\lim _{n \rightarrow \infty} M\left(x_{n+p}, x_{n}, t\right)=1
$$

for all $t>0$ and $p>0$.

(3) An FM-space in which every Cauchy sequence is convergent is said to be complete. 
REMARK 1 . Since $*$ is continuous, it follows from (fm- 4 ) that the limit of sequence in FM-space is uniquely determined.

Throughout this paper $(X, M, *)$ will denote the fuzzy metric space with the following condition:

(fm-6): $\lim _{t \rightarrow \infty} M(x, y, t)=1$ for all $x, y \in X$ and $t>0$.

LEMMA 1. (Cho [2] and Mishra et al. [19]). Let $\left\{y_{n}\right\}$ be a sequence in an $F M$-space $(X, M, *)$ with the condition (fm-6). If there is a number $k \in(0,1)$ such that

$$
M\left(y_{n+2}, y_{n+1}, k t\right) \geq M\left(y_{n+1}, y_{n}, t\right)
$$

for all $t>0$ and $n=1,2 \ldots$, then $\left\{y_{n}\right\}$ is a Cauchy sequence in $X$.

\section{Compatible maps of type $(\beta)$}

In this section, we give the concept of compatible maps of type $(\beta)$ in FM- spaces and some properties of these maps.

The notion of compatible maps of type $(\beta)$ in FM-space $(X, M, *)$ was first introduced by Cho et al. [3]. The condition " $a * a \geq a$ for all $a \in[0,1]$ " in properties given by Cho et al. [3] did not play an essential role in proof of our main results. So, we give the properties of compatible maps of type $(\beta)$ in fuzzy metric spaces with arbitrary continuous t-norms. More details we refer to reader $[10,16,17]$.

Definition 4. (Mishra et al. [19]). Let $A$ and $B$ be maps from an FM-space $(X, M, *)$ into itself. The maps $A$ and $B$ are said to be compatible if

$$
\lim _{n \rightarrow \infty} M\left(A B x_{n}, B A x_{n}, t\right)=1
$$

for all $t>0$ whenever $\left\{x_{n}\right\}$ is a sequence in $X$ such that

$$
\lim _{n \rightarrow \infty} A x_{n}=\lim _{n \rightarrow \infty} B x_{n}=z
$$

for some $z \in X$.

Definition 5. (Cho et al. [3]). Let $A$ and $B$ be maps from an FMspace $(X, M, *)$ into itself. The maps $A$ and $B$ are said to be compatible of type $(\beta)$ if

$$
\lim _{n \rightarrow \infty} M\left(A A x_{n}, B B x_{n}, t\right)=1
$$

for all $t>0$ whenever $\left\{x_{n}\right\}$ is a sequence in $X$ such that

for some $z \in X$.

$$
\lim _{n \rightarrow \infty} A x_{n}=\lim _{n \rightarrow \infty} B x_{n}=z
$$


REMARK 2. In [14, 20], we can find the equivalent; formulations of Definition 4 and 5 and their examples in metric spaces. Such maps are independent of each other and more general than commuting and weakly commuting maps $[13,24]$.

Proposition 1. Let $(X, M, *)$ be an $F M$-space and $A, B$ be continuous maps from $X$ into itself. Then $A$ and $B$ are compatible if and only if they are compatible of type $(\beta)$.

Proposition 2. Let $(X, M, *)$ be an FM-space and $A, B$ be maps from $X$ into itself. If $A$ and $B$ are compatible of type $(\beta)$ and $A z=B z$ for some $z \in X$, then $A B z=B B z=B A z=A A z$.

Proposition 3. Let $(X, M, *)$ be an FM-space and $A, B$ be compatible maps of type $(\beta)$ from $X$ into itself. Let $\left\{x_{n}\right\}$ be a sequence in $X$ such that

$$
\lim _{n \rightarrow \infty} A x_{n}=\lim _{n \rightarrow \infty} B x_{n}=z
$$

for some $z \in X$. Then we have the following:

(i) $\lim _{n \rightarrow \infty} B B x_{n}=A z$ if $A$ is continuous at $z$,

(ii) $\lim _{n \rightarrow \infty} A A x_{n}=B z$ if $B$ is continuous at $z$,

(iii) $A B z=B A z$ and $A z=B z$ if $A$ and $B$ are continuous at $z$.

EXAMPLE 1 . Let the set $X=[0, \infty)$ with the metric $d$ defined by $d(x, y)=|x-y|$ and for each $t>0$ define $M(x, y, t)=\frac{t}{t+d(x, y)}$ for all $x, y \in X$. Clearly $(X, M, *)$ is a fuzzy metric space where $*$ is defined by $a * b=a b$. Define $A, B: X \rightarrow X$ by $A x=1$ for $x \in[0,1], A x=$ $1+x$ for $x \in(1, \infty)$, and $B x=1+x$ for $x \in[0,1), B x=1$ for $x \in[1, \infty)$. Then $A$ and $B$ both are discontinuous at $x=1$. Consider the sequence $\left\{x_{n}\right\}$ in $X$ defined by $x_{n}=1 / n, n=1,2, \ldots$. Then we have $\lim _{n} A x_{n}=\lim _{n} B x_{n}=1$. Further, $\lim _{n} M\left(A B x_{n}, B A x_{n}, t\right) \neq 1$ and $\lim _{n} M\left(A A x_{n}, B B x_{n}, t\right)=1$. Therefore $A$ and $B$ are compatible of type $(\beta)$ but they are not compatible.

EXAMPLE 2 . Let the set $X=\mathbb{R}$ with the metric $d$ defined by $d(x, y)=$ $|x-y|$ and for each $t>0$ define $M(x, y, t)=\frac{t}{t+d(x, y)}$ for all $x, y \in X$. Clearly $(X, M, *)$ is a fuzzy metric space where $*$ is defined by $a * b=a b$. Define $A, B: X \rightarrow X$ by $A x=1 / x^{3}$ for $x \neq 0, A x=1$ for $x=0$, and $B x=1 / x^{2}$ for $x \neq 0, B x=2$ for $x=0$. Then $A$ and $B$ both are discontinuous at $x=0$. Consider the sequence $\left\{x_{n}\right\}$ in $X$ defined by $x_{n}=n, n=1,2, \ldots$. Then we have $\lim _{n} A x_{n}=\lim _{n} B x_{n}=0$. Further, $\lim _{n} M\left(A B x_{n}, B A x_{n}, t\right)=1$ and $\lim _{n} M\left(A A x_{n}, B B x_{n}, t\right)=0$. 
Therefore $A$ and $B$ are compatible but they are not compatible of type $(\beta)$.

\section{Main results}

THEOREM 1. Let $(X, M, *)$ be a complete $F M$-space and let $P, S, T$ and $Q$ be maps from $X$ into itself such that

(1) $P T(X) \cup Q S(X) \subset S T(X)$,

(2) there exists a constant $k \in(0,1)$ such that

$$
\begin{aligned}
& M^{2}(P x, Q y, k t) *[M(S x, P x, k t) M(T y, Q y, k t)] * M^{2}(T y, Q y, k t) \\
& +a M(T y, Q y, k t) M(S x, Q y, 2 k t) \\
\geq & {[p M(S x, P x, t)+q M(S x, T y, t)] M(S x, Q y, 2 k t) }
\end{aligned}
$$

for all $x, y$ in $X$ and $t>0$ where $0<p, q<1,0 \leq a<1$ such that $p+q-a=1$,

(3) $S$ and $T$ are continuous and $S T=T S$,

(4) the pairs $P, S$ and $Q, T$ are compatible of type $(\beta)$.

Then $P, S, T$ and $Q$ have unique common fixed point in $X$.

Proof. Let $x_{0}$ be an arbitrary point of $X$. By (1), we can construct a sequence $\left\{x_{n}\right\}$ in $X$ as follows:

$$
P T x_{2 n}=S T x_{2 n+1}, Q S x_{2 n+1}=S T x_{2 n+2}, n=0,1,2, \ldots
$$

Indeed, such a sequence was first introduced in [26, 27].

Now, let $z_{n}=S T x_{n}$. Then, by (2), we have

$$
\begin{aligned}
& M^{2}\left(P T x_{2 n}, Q S x_{2 n+1}, k t\right) *\left[M\left(S T x_{2 n}, P T x_{2 n}, k t\right)\right. \\
& \left.M\left(T S x_{2 n+1}, Q S x_{2 n+1}, k t\right)\right] * M^{2}\left(T S x_{2 n+1}, Q S x_{2 n+1}, k t\right) \\
& +a M\left(T S x_{2 n+1}, Q S x_{2 n+1}, k t\right) M\left(S T x_{2 n}, Q S x_{2 n+1}, 2 k t\right) \\
\geq & {\left[p M\left(S T x_{2 n}, P T x_{2 n}, t\right)+q M\left(S T x_{2 n}, T S x_{2 n+1}, t\right)\right] } \\
& M\left(S T x_{2 n}, Q S x_{2 n+1}, 2 k t\right)
\end{aligned}
$$

and

$$
\begin{aligned}
& M^{2}\left(S T x_{2 n+1}, S T x_{2 n+2}, k t\right) *\left[M\left(z_{2 n}, S T x_{2 n+1}, k t\right)\right. \\
& \left.M\left(z_{2 n+1}, S T x_{2 n+2}, k t\right)\right] * M^{2}\left(z_{2 n+1}, S T x_{2 n+2}, k t\right) \\
& +a M\left(z_{2 n+1}, S T x_{2 n+2}, k t\right) M\left(z_{2 n}, S T x_{2 n+2}, 2 k t\right) \\
\geq & {\left[p M\left(z_{2 n}, S T x_{2 n+1}, t\right)+q M\left(z_{2 n}, z_{2 n+1}, t\right)\right] M\left(z_{2 n}, S T x_{2 n+2}, 2 k t\right) }
\end{aligned}
$$


then

$$
\begin{aligned}
& M^{2}\left(z_{2 n+1}, z_{2 n+2}, k t\right) *\left[M\left(z_{2 n}, z_{2 n+1}, k t\right) M\left(z_{2 n+1}, z_{2 n+2}, k t\right)\right] \\
& * M^{2}\left(z_{2 n+1}, z_{2 n+2}, k t\right)+a M\left(z_{2 n+1}, z_{2 n+2}, k t\right) M\left(z_{2 n}, z_{2 n+2}, 2 k t\right) \\
\geq & {\left[p M\left(z_{2 n}, z_{2 n+1}, t\right)+q M\left(z_{2 n}, z_{2 n+1}, t\right)\right] M\left(z_{2 n}, \varkappa_{2 n+2}, 2 k t\right) }
\end{aligned}
$$

so

$$
\begin{aligned}
& M^{2}\left(z_{2 n+1}, z_{2 n+2}, k t\right) *\left[M\left(z_{2 n}, z_{2 n+1}, k t\right) M\left(z_{2 n+1}, z_{2 n+2}, k t\right)\right] \\
& +a M\left(z_{2 n+1}, z_{2 n+2}, k t\right) M\left(z_{2 n}, z_{2 n+2}, 2 k t\right) \\
\geq & {[p+q] M\left(z_{2 n}, z_{2 n+1}, t\right) M\left(z_{2 n}, z_{2 n+2}, 2 k t\right) }
\end{aligned}
$$

and

$$
\begin{aligned}
& M\left(z_{2 n+1}, z_{2 n+2}, k t\right)\left[M\left(z_{2 n}, z_{2 n+1}, k t\right) * M\left(z_{2 n+1}, z_{2 n+2}, k t\right)\right] \\
& +a M\left(z_{2 n+1}, z_{2 n+2}, k t\right) M\left(z_{2 n}, z_{2 n+2}, 2 k t\right) \\
\geq & {[p+q] M\left(z_{2 n}, z_{2 n+1}, t\right) M\left(z_{2 n}, z_{2 n+2}, 2 k t\right) }
\end{aligned}
$$

and

$$
\begin{aligned}
& M\left(z_{2 n+1}, z_{2 n+2}, k t\right) M\left(z_{2 n}, z_{2 n+2}, 2 k t\right) \\
& +a M\left(z_{2 n+1}, z_{2 n+2}, k t\right) M\left(z_{2 n}, z_{2 n+2}, 2 k t\right) \\
\geq \quad & {[p+q] M\left(z_{2 n}, z_{2 n+1}, t\right) M\left(z_{2 n}, z_{2 n+2}, 2 k t\right) . }
\end{aligned}
$$

Thus, it follows that

$$
M\left(z_{2 n+1}, z_{2 n+2}, k t\right) \geq M\left(z_{2 n}, z_{2 n+1}, t\right)
$$

$0<k<1$ and for all $t>0$.

Similarly, we also have

$$
M\left(z_{2 n+2}, z_{2 n+3}, k t\right) \geq M\left(z_{2 n+1}, z_{2 n+2}, t\right)
$$

$0<k<1$ and for all $t>0$.

In general, for $m=1,2, \ldots$, we have

$$
M\left(z_{m+1}, z_{m+2}, k t\right) \geq M\left(z_{m}, z_{m+1}, t\right)
$$

$0<k<1$ and for all $t>0$. Hence, by Lemma 1, $\left\{z_{n}\right\}$ is a Cauchy sequence in $X$. Since $(X, M, *)$ is complete, it converges to a point $z$ in $X$. Since $\left\{P T x_{2 n}\right\}$ and $\left\{Q S x_{2 n+1}\right\}$ are subsequences of $\left\{z_{n}\right\}, P T x_{2 n} \rightarrow$ $z$ and $Q S x_{2 n+1} \rightarrow z$ as $n \rightarrow \infty$.

Let $y_{n}=T x_{n}$ and $w_{n}=S x_{n}$ for $n=1,2, \ldots$ Then, we have $P y_{2 n} \rightarrow$ $z, S y_{2 n} \rightarrow z, T w_{2 n+1} \rightarrow z$ and $Q w_{2 n+1} \rightarrow z$,

$$
M\left(P P y_{2 n}, S S y_{2 n}, t\right) \rightarrow 1 \text { and } M\left(Q Q w_{2 n+1}, T T w_{2 n+1}, t\right) \rightarrow 1
$$


as $n \rightarrow \infty$. Moreover, by the continuity of $T$ and Proposition 3 , we have

$$
T Q w_{2 n+1} \rightarrow T z \text { and } Q Q w_{2 n+1} \rightarrow T z
$$

as $n \rightarrow \infty$. Now, taking $x=y_{2 n}$ and $y=Q w_{2 n+1}$ in (2), we have

$$
\begin{aligned}
& M^{2}\left(P y_{2 n}, Q Q w_{2 n+1}, k t\right) *\left[M\left(S y_{2 n}, P y_{2 n}, k t\right)\right. \\
& \left.M\left(T Q w_{2 n+1}, Q Q w_{2 n+1}, k t\right)\right] * M^{2}\left(T Q w_{2 n+1}, Q Q w_{2 n+1}, k t\right) \\
& +a M\left(T Q w_{2 n+1}, Q Q w_{2 n+1}, k t\right) M\left(S y_{2 n}, Q Q w_{2 n+1}, 2 k t\right) \\
\geq & {\left[p M\left(S y_{2 n}, P y_{2 n}, t\right)\right.} \\
& \left.+q M\left(S y_{2 n}, T Q w_{2 n+1}, t\right)\right] M\left(S y_{2 n}, Q Q w_{2 n+1}, 2 k t\right)
\end{aligned}
$$

and

$$
\begin{aligned}
& M^{2}(z, T z, k t) *[M(z, z, k t) M(T z, T z, k t)] * M^{2}(T z, T z, k t) \\
& +a M(T z, T z, k t) M(z, T z, 2 k t) \\
\geq & {[p M(z, z, t)+q M(z, T z, t)] M(z, T z, 2 k t) }
\end{aligned}
$$

then, it follows that

$$
M^{2}(z, T z, k t)+a M(z, T z, 2 k t) \geq[p+q M(z, T z, t)] M(z, T z, 2 k t)
$$

and since $M(x, y,$.$) is non-decreasing for all x, y$ in $X$, we have

$$
\begin{aligned}
& M(z, T z, 2 k t) M(z, T z, t)+a M(z, T z, 2 k t) \\
\geq & {[p+q M(z, T z, t)] M(z, T z, 2 k t) }
\end{aligned}
$$

thus

$$
M(z, T z, t)+a \geq p+q M(z, T z, t)
$$

and

$$
M(z, T z, t) \geq \frac{p-a}{1-q}=1
$$

for all $t>0$ so $z=T z$. Similarly, we have $z=S z$.

Now, taking $x=y_{2 n}$ and $y=z$ in (2), we have

$$
\begin{aligned}
& M^{2}\left(P y_{2 n}, Q z, k t\right) *\left[M\left(S y_{2 n}, P y_{2 n}, k t\right) M(T z, Q z, k t)\right] \\
& * M^{2}(T z, Q z, k t)+a M(T z, Q z, k t) M\left(S y_{2 n}, Q z, 2 k t\right) \\
\geq & {\left[p M\left(S y_{2 n}, P y_{2 n}, t\right)+q M\left(S y_{2 n}, T z, t\right)\right] M\left(S y_{2 n}, Q z, 2 k t\right) }
\end{aligned}
$$

and

$$
\begin{aligned}
& M^{2}(z, Q z, k t) *[M(z, z, k t) M(z, Q z, k t)] * M^{2}(z, Q z, k t) \\
& +a M(z, Q z, k t) M(z, Q z, 2 k t) \\
\geq & {[p M(z, z, t)+q M(z, z, t)] M(z, Q z, 2 k t) }
\end{aligned}
$$


then

$$
\begin{aligned}
& M^{2}(z, Q z, k t) * M(z, Q z, k t)+a M(z, Q z, k t) M(z, Q z, 2 k t) \\
\geq \quad & (p+q) M(z, Q z, 2 k t)
\end{aligned}
$$

so

$$
\begin{aligned}
& M(z, Q z, k t)[M(z, Q z, k t) * 1]+a M(z, Q z, k t) M(z, Q z, 2 k t) \\
\geq \quad & (p+q) M(z, Q z, 2 k t)
\end{aligned}
$$

and since $M(x, y,$.$) is non-decreasing for all x, y$ in $X$, we have

$$
\begin{aligned}
& M(z, Q z, 2 k t) M(z, Q z, k t)+a M(z, Q z, k t) M(z, Q z, 2 k t) \\
\geq \quad & (p+q) M(z, Q z, 2 k t) .
\end{aligned}
$$

Thus it follows that

$$
M(z, Q z, k t)+a M(z, Q z, k t) \geq p+q
$$

and

$$
M(z, Q z, k t) \geq \frac{p+q}{1+a}=1
$$

$0<k<1$ and for all $t>0$ so $z=Q z$. Similarly, we have $z=P z$. Therefore, $z$ is a common fixed point of $P, Q, S$ and $T$.

Let $v$ be second common fixed point of $P, Q, S$ and $T$. Then using inequality (2), we have

$$
\begin{aligned}
& M^{2}(P z, Q v, k t) *[M(S z, P z, k t) M(T v, Q v, k t)] * M^{2}(T v, Q v, k t) \\
& +a M(T v, Q v, k t) M(S z, Q v, 2 k t) \\
\geq & {[p M(S z, P z, t)+q M(S z, T v, t)] M(S z, Q v, 2 k t) }
\end{aligned}
$$

so

$$
M^{2}(z, v, k t)+a M(z, v, 2 k t) \geq[p+q M(z, v, t)] M(z, v, 2 k t)
$$

and

$$
M(z, v, t) M(z, v, 2 k t)+a M(z, v, 2 k t) \geq[p+q M(z, v, t)] M(z, v, 2 k t) .
$$

Thus, it follows that

$$
M(z, v, t) \geq \frac{p-a}{1-q}=1
$$

for all $t>0$ so $z=v$. Hence $P, S, T$ and $Q$ have unique common fixed point.

If we put $a=0$ in Theorem 1, we have the following result: 
Corollary 1. Let $(X, M, *)$ be a complete $F M$-space and let $P, S$, $T$ and $Q$ be maps from $X$ into itself such that the conditions (1), (3) and (4) of the Theorem 1 hold and there exists a constant $k \in(0,1)$ such that

$$
\begin{aligned}
& M^{2}(P x, Q y, k t) *[M(S x, P x, k t) M(T y, Q y, k t)] * M^{2}(T y, Q y, k t) \\
\geq & {[p M(S x, P x, t)+q M(S x, T y, t)] M(S x, Q y, 2 k t) }
\end{aligned}
$$

for all $x, y$ in $X$ and $t>0$ where $0<p, q<1$ such that $p+q=1$. Then $P, S, T$ and $Q$ have unique common fixed point in $X$.

If we put $S=T$ in Theorem 1, we have the following result:

Corollary 2. Let $(X, M, *)$ be a complete $F M$-space and let $P, S$ and $Q$ be maps from $X$ into itself such that

(1) $P(X) \cup Q(X) \subset S(X)$,

(2) there exists a constant $k \in(0,1)$ such that

$$
\begin{aligned}
& M^{2}(P x, Q y, k t) *[M(S x, P x, k t) M(S y, Q y, k t)] * M^{2}(S y, Q y, k t) \\
& +a M(S y, Q y, k t) M(S x, Q y, 2 k t) \\
\geq & {[p M(S x, P x, t)+q M(S x, S y, t)] M(S x, Q y, 2 k t) }
\end{aligned}
$$

for all $x, y$ in $X$ and $t>0$ where $0<p, q<1,0 \leq a<1$ such that $p+q-a=1$,

(3) $S$ is continuous,

(4) the pairs $P, S$ and $Q, S$ are compatible of type $(\beta)$.

Then $P, S$ and $Q$ have unique common fixed point in $X$.

If we put $S=T$ and $P=Q$ in Theorem 1, we have the following result:

Corollary 3. Let $(X, M, *)$ be a complete $F M$-space and let $S$ and $P$ be maps from $X$ into itself such that

(1) $P(X) \subset S(X)$

(2) there exists a constant $k \in(0,1)$ such that

$$
\begin{aligned}
& M^{2}(P x, P y, k t) *[M(S x, P x, k t) M(S y, P y, k t)] * M^{2}(S y, P y, k t) \\
&+a M(S y, P y, k t) M(S x, P y, 2 k t) \\
& \geq {[p M(S x, P x, t)+q M(S x, S y, t)] M(S x, P y, 2 k t) } \\
& \text { for all } x, y \text { in } X \text { and } t>0 \text { where } 0<p, q<1,0 \leq a<1 \text { such that } \\
& p+q-a=1, \\
& \text { (3) } S \text { is continuous, } \\
& \text { (4) } P \text { and } S \text { are compatible of type }(\beta) .
\end{aligned}
$$


Then $P$ and $S$ have unique common fixed point in $X$.

If we put $S=T=I_{X}$ (the identity map on $X$ ) in Theorem 1, we have the following result:

Corollary 4. Let $(X, M, *)$ be a complete $F M$-space and let $P, Q$ be maps from $X$ into itself. If there exists a constant $k \in(0,1)$ such that

$$
\begin{aligned}
& M^{2}(P x, Q y, k t) *[M(x, P x, k t) M(y, Q y, k t)] * M^{2}(y, Q y, k t) \\
& +a M(y, Q y, k t) M(x, Q y, 2 k t) \\
\geq & {[p M(x, P x, t)+q M(x, y, t)] M(x, Q y, 2 k t) }
\end{aligned}
$$

for all $x, y$ in $X$ and $t>0$ where $0<p, q<1,0 \leq a<0$ such that $p+q-a=1$. Then $P$ and $Q$ have unique common fixed point in $X$.

The following example illustrates our main Theorem.

EXAMPle 3. Let $X=\left\{\frac{1}{n}: n \in \mathbb{N}\right\} \cup\{0\}$ with the metric $d$ defined by $d(x, y)=|x-y|$ and for each $t>0$ define $M(x, y, t)=\frac{t}{t+d(x, y)}$ for all $x, y \in X$. Clearly $(X, M, *)$ is a complete fuzzy metric space where $*$ is defined by $a * b=a b$. Let $P, S, T$ and $Q$ be maps from $X$ into itself defined as

$$
P x=\frac{x}{4}, S x=\frac{x}{2}, T x=x, Q x=0
$$

for all $x \in X$. Then

$P T(X) \cup Q S(X)=\left\{\frac{1}{4 n}: n \in \mathbb{N}\right\} \cup\{0\} \subset\left\{\frac{1}{2 n}: n \in \mathbb{N}\right\} \cup\{0\}=S T(X)$.

Clearly $S T=T S$ and $S, T$ are continuous. If we take $k=\frac{1}{2}$ and $t=1$, we see that the condition (2) of the main Theorem is also satisfied. Moreover, the maps $P$ and $S$ are compatible of type $(\beta)$ if $\lim _{n \rightarrow \infty} x_{n}=0$, where $\left\{x_{n}\right\}$ is a sequence in $X$ such that $\lim _{n \rightarrow \infty} P x_{n}==\lim _{n \rightarrow \infty} S x_{n}=0$ for some $0 \in X$. Similarly, the maps $Q$ and $T$ are also compatible of type $(\beta)$. Thus, all the conditions of main Theorem are satisfied and 0 is the unique common fixed point of $P, S, T$ and $Q$.

\section{References}

[1] S. Banach, Theorie les operations lineaires, Manograie Mathematyezne, Warsaw, Poland, 1932.

[2] Y. J. Cho, Fixed points in fuzzy metric spaces, J. Fuzzy Math. 4 (1997), 949-962.

[3] Y. J. Cho, H. K. Pathak, S. M. Kang, and J. S. Jung, Common fixed points of compatible maps of type $(\beta)$ on fuzzy metric spaces, Fuzzy Sets and Systems $\mathbf{9 3}$ (1998), 99-111.

[4] Z. K. Deng, Fuzzy pseduo-metric spaces, J. Math. Anal. Appl. 86 (1982), 74-95. 
[5] M. Edelstein, On fixed and periodic points under contraction mappings, J. London Math. Soc. 37 (1962) 74-79.

[6] M. A. Erceg, Metric spaces in fuzzy set theory, J. Math. Anal. Appl. 69 (1979), 205-230.

[7] J. X. Fang, On fixed point theorems in fuzzy metric spaces, Fuzzy Sets and Systems 46 (1992), 107-113.

[8] A. George and P. Veeramani, On some results in fuzzy metric spaces, Fuzzy Sets and Systems 64 (1994), 395-399.

[9] M. Grabiec, Fixed points in fuzzy metric space, Fuzzy Sets and Systems 27 (1988), 385-389.

[10] O. Hadzic and E. Pap, Fixed point theory in probabilistic metric spaces, Kluwer Acad. Publ., 2001.

[11] _ , A fixed point theorem for multivalued mappins in probabilistic metric spaces and an application in fuzzy metric spaces, Fuzzy Sets and Systems $\mathbf{1 2 7}$ (2002), 333-344.

[12] I. Istratescu, A fixed point theorem for mappings with a probabilistic contractive iterate, Rev. Roumaire. Math. Pure Appl, 26 (1981), 431-435.

[13] G. Jungck, Commuting mappings and fixed points, Amer. Math. Monthly 83 (1976), 261-263.

[14] Compatible mappings and common fixed points, Internat. J. Math. Math. Sci. 9 (1986), 771-779.

[15] O. Kaleva and S. Seikkala, On fuzzy metric spaces, Fuzzy Sets and Systems 12 (1984), 215-229.

[16] E. P. Klement, R. Mesiar, and E. Pap, Triangular norms. Position paper I: Basic analytical and algebraic properties, Fuzzy Sets and Systems 143 (2004), 5-16.

[17] _ Problems on triangular norms and related operators, Fuzzy Sets and Systems 145 (2004), 471-479.

[18] O. Kramosil and J. Michalek, Fuzzy metric and statistical metric spaces, Kybernetika (Praha) 11 (1975), 326-334.

[19] S. N. Mishra, N. Sharma, and S. L. Singh, Common fixed points of maps on fuzzy metric spaces, Internat. J. Math. Sci. 17 (1994), 253-258.

[20] H. K. Pathak, Y. J. Cho, S. S. Chang, and S. M. Kang, Compatible mappings of type (P), Rev. Res. Univ. Novi Sad., to appear.

[21] B. E. Rhoades, A comparison of various definitions of contractive mappings, Trans. Amer. Math. Soc. 226 (1977), 257-290.

[22] B. Schweizer and A. Sklar, Statistical metric spaces, Pacific J. Math. 10 (1960), 313-334.

[23] V. M. Sehgal and A. T. Bharucha-Reid, Fixed point of contraction mapping on PM spaces, Math. Systems Theory 6 (1972), 97-100.

[24] S. Sessa, On weak commutativity condition of mappings in fixed point considerations, Publ. Inst. Math. Beagrad 32 (46) (1982), 149-153.

[25] S. Sharma, Common Fixed point theorems in fuzzy metric spaces, Fuzzy Sets and Systems 127 (2002), 345-352.

[26] S. L. Singh and S. Kasahara, On some recent results on common fixed points, Indian J. Pure Appl. Math. 13 (1982), 757-761.

[27] S. L. Singh and B. Ram, Common fixed points of commuting mappings in 2metric spaces, Math. Sem. Notes Kobe Univ. 10 (1982), 197-208. 
[28] L. A. Zadeh, Fuzzy sets, Inform. and Control 8 (1965), 338-353

Department of Mathematics

Faculty of Science and Arts

University of Gazi

06500 Ankara, Turkey

E-mail: servet@gazi.edu.tr dturkoglu@gazi.edu.tr

cyildiz@gazi.edu.tr 